\title{
Depression Detection Using Virtual Reality: A Literature Review
}

\author{
Chi Zhang \\ Kennesaw State University \\ chizhang@kennesaw.edu
}

\author{
Hao (Irene) Luo \\ Renmin University of China \\ irenehao.luo@gmail.com
}

\author{
Yi (Joy) Li \\ Kennesaw State University \\ joy.li@kennesaw.edu
}

\begin{abstract}
Depression is a mood disorder that can cause mental suffering, functional impairment, physical abuse or automutilation. Early detection will help depressed people to seek professional advice and treatment earlier and recover sooner. Emerging technologies such as virtual reality (VR) have been used to assist in interventions because of their advantages in providing more flexible solutions to implement therapeutic methods. However, the applications of using VR as a diagnostic tool for mental disorders have not been thoroughly investigated.

In this study, prior studies that used VR for detecting depression were reviewed and summarized. Also reviewed were other emerging technologies for depression detection. By summarizing the effort on this topic and proposing an integrated framework, we hope that the presented work will reduce the barriers to implement emerging technologies in supporting traditional depression diagnostic methods, with a further expectation to provide inspiration and guidance for future studies.
\end{abstract}

\section{Introduction}

The COVID-19 pandemic can be stressful for people and has raised the concern about public mental health [1]. The stress and anxiety caused by either the disease or the financial situation could cause overwhelmingly strong emotions, and even depression [2, 3]. In fact, among the most prevalent psychiatric disorders, major depressive disorder, also referred to as clinical depression, is affecting more than 300 million people globally [4]. The traditional methods used to diagnose depression mainly include scale examination, blood test, and interviews with professional medical staff, but the spread of COVID-19 also makes it difficult for conventional face-to-face interviews to take place. In some cases, people who suffer from depression may not be willing to reach out and seek help from professionals, and thus remain untreated. As early detection has been determined to be critical for rapid intervention to reduce the escalation of major depressive disorder cases $[5,6$, 7], serious games that aim to effectively identify the depressive symptoms and detect potential depression in a relaxed and non-intrusive form can be beneficial. It helps potential patients who are unable or reluctant to face the pressure of interaction with a real person. As emerging gaming tools including mixed realities, eye tracking, and smart wearable devices, enter the mainstream, virtual reality (VR) has become more accessible to the general consumer population, and has been studied how it helps to treat mental disorders $[8,9$, 10]. In most cases, these emerging technologies are used as supplementary methods for therapies or interventions. They provide non-intrusive and immersive experiences in cost-effective ways for the treatment of mental illness. However, not many studies have addressed the use of emerging technologies for assisting symptom selfdetection, or the importance of early detection and intervention, especially in children and adolescence [11, 12]. This motivated us to conduct a literature review to summarize the current state of VR game design for depression detection as preparation to propose our game design and study plan as the next step of this research.

In this paper, we reviewed the studies that made use of virtual reality (not limited to interactive games) to assist the detection or diagnosis of depression. Based on the findings, we extended the review scope to investigate other methodologies for depression detection in the past ten years. Finally, we proposed an integrated framework for future studies.

\section{Background}

Previous studies have discussed that VR provides a fully immersive experience $[13,14,15,16,17]$ and can meet the high requirement on variable controls in psychology and neuroscience research [18]. The possibility of triggering stronger and more specific emotional reactions in a safer environment has motivated the interest to use VR as a therapeutic intervention for patients with mental disorders such as depression [19]. There are systematic reviews and metaanalysis papers that summarized VR-assisted therapies for mental disorders and their efficiency in recent years $[20,21,22,23]$.

Depressive disorder, one of the leading causes of psychiatric disability worldwide [24], can evoke severe symptoms that impede the daily activities of patients and how they feel and think. There are known evidence 
of deficits associated with depression, for example, social skills deficits [25], cognitive deficits [26], executive deficits [27], memory deficits [28] and more. Some of the symptoms and deficits can be targeted as the indicators in assisting early detection of depression, such as visual indicators, speech indicators, social indicators, among others [29]. Thus, they can also be used in the interactive narratives of games. VR applications include VR 360 videos, simulations, and games with interactive narratives. While VR 360 videos provide immersive experiences, simulations mockup provides specific real-world scenarios. Additionally, interactive games are able to embed the tasks into storytelling [30], so users can receive instant feedback and become aware of the consequences of their actions. This leads to the advantages of interactive VR applications as users can experience more effective observation and emotional elicitation, and thereby a stronger presence and better immersion. As a result, fully interactive VR games engage players in scenarios which enable them to disclose information with relaxation and show more bahavioral and emotional reactions than videos or simulations. Although more professional measurements are still pending for a formal diagnosis, early detection of depression via gaming can be more motivational and helpful to alert users if they have high risks and need to seek professional treatment.

\section{Materials and methods}

In this section, we describe the procedure and results from the review of designing detection methods using VR for depression. The PRISMA Statement [31] served as a reference for the review summary.

\subsection{Information Sources and Search Strategy}

Eight databases, Google Scholar, Crossref, PubMed, Science Direct, IEEE, SpringerLink, Wiley, Tylor \& Francis, were searched for publications between 20102020. The time period was determined because most of the head-mounted virtual reality (VR) devices are based on Oculus Rift Development Kit 1, an open-source VR system for developing virtual reality games, which was not available until mid-2013 [32]. This research aims to find the studies related to using virtual reality for depression detection over the past decade as reference for future research directions. The key search term was: "depress*" in the title and "virtual reality" in keywords. Furthermore, "detect/screen/diagn*" was used to find eligible papers to identify the games for depression detection in the preliminary results. The references in the included articles were also screened for additional qualified studies. In order to discuss the methodologies used in future VR game design, we included additional search results of general depression detection methods.

One researcher (Author YL) performed the search and identification of qualified studies. Two other researchers (Authors HL and CZ) cross-checked on the classification and assessment of the paper quality. They also participated in discussions when there was disagreement in the inclusion or exclusion of the papers for the review.

\subsection{Eligibility Criteria}

The review included articles that are:

- With full text written in English only;

- Addressing depression or depression-related mental disorders;

- Using some form of mixed reality as one of the methods, including non-immersive VR videos or augmented reality (AR);

- about depression detection, including early screening or early diagnosis.

\section{Results}

\subsection{Study Selection Results}

The search yielded 427 potentially relevant articles, and 438 additional articles were identified through references or extended scope (refer to subsection 4.5). Figure 1 shows the PRISMA flow chart of the search process and the numbers of inclusion and exclusion at each step. Five studies that focuses on using VR/AR to detect depression were included in the review summary in Table 1. An additional 31 studies were included in the extended review as explained in 4.5. These 31 studies discussed how technology-based methods had been used for depression detections in the past ten years.

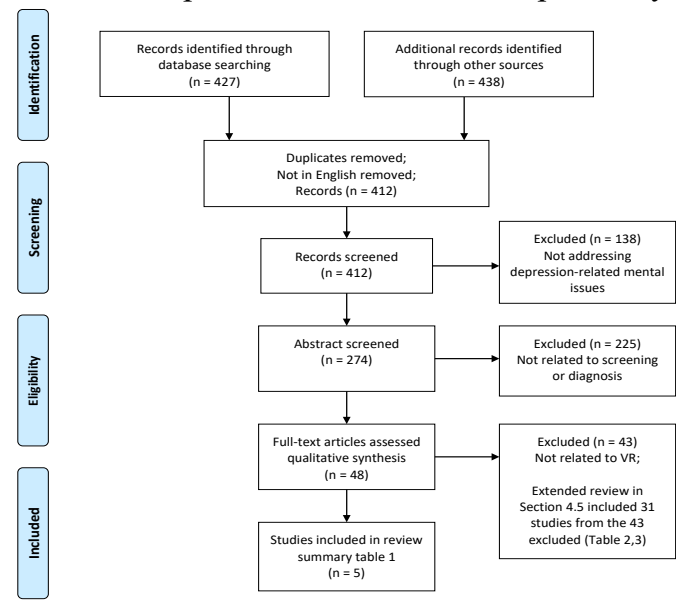

Figure 1. The PRISMA Search Process 
Table 1. Summary of Findings

\begin{tabular}{|c|c|c|c|c|c|}
\hline $\begin{array}{c}\text { Refer } \\
\text { ence }\end{array}$ & Methods & Participants & Experiments & Outcomes & Notes \\
\hline [33] & $\begin{array}{l}\text { A virtual human interviewer was } \\
\text { designed to create an engaging } \\
\text { face-to-face interaction to } \\
\text { identify depression, anxiety or } \\
\text { PTSD through verbal and } \\
\text { gestural responses. }\end{array}$ & $\mathrm{N}=351$ & $\begin{array}{l}\text { Three groups: face-to-face; virtual } \\
\text { puppet controlled by human; } \\
\text { complete AI interaction. } \\
\text { Self-report was given pre- and post- } \\
\text { experience. }\end{array}$ & $\begin{array}{l}\text { Participants reported willingness to } \\
\text { disclose, willingness to recommend, and } \\
\text { general satisfaction with both the semi } \\
\text { and AI versions of the system. }\end{array}$ & $\begin{array}{l}\text { System Usability } \\
\text { Scale was used for } \\
\text { assessment of system } \\
\text { satisfaction and } \\
\text { usability. } \\
\text { Jadad scale }=3\end{array}$ \\
\hline$[34]$ & $\begin{array}{l}\text { Performance on a novel virtual } \\
\text { reality navigation task and a } \\
\text { traditional measure of spatial } \\
\text { memory was assessed. T-tests } \\
\text { and Pearson correlations were } \\
\text { used. All statistics were } \\
\text { evaluated at } \mathrm{p} \leq 0.05 \text {, two-tailed. }\end{array}$ & $\begin{array}{l}\mathrm{N}=40 \\
30 \text { depressed } \\
\text { patients; } \\
10 \text { control } \\
\text { subjects }\end{array}$ & $\begin{array}{l}\text { A VR Town Represented in Spatial } \\
\text { Memory Navigation. } \\
\text { Two tasks were evaluated: finding } \\
\text { locations on the navigation tasks, and } \\
\text { Spatial Working Memory task. }\end{array}$ & $\begin{array}{l}\text { Depressed patients performed } \\
\text { significantly worse than the control } \\
\text { group on the virtual reality task, as } \\
\text { assessed by the number of locations } \\
\text { found in the virtual town. Between- } \\
\text { group differences were not detected on } \\
\text { the traditional measure. The navigation } \\
\text { task showed high test-retest reliability. }\end{array}$ & $\begin{array}{l}\text { Virtual reality } \\
\text { navigation may } \\
\text { provide a consistent, } \\
\text { sensitive measure of } \\
\text { cognitive deficits in } \\
\text { patients with } \\
\text { affective disorders. } \\
\text { Jadad scale }=5\end{array}$ \\
\hline$[35]$ & $\begin{array}{l}\text { The study investigated the } \\
\text { relationship between EEG } \\
\text { signals and depression under } \\
\text { induced emotions using VR. }\end{array}$ & $\begin{array}{l}\mathrm{N}=463 ; \\
55 \text { identified } \\
\text { as high risk } \\
\text { of } \\
\text { depression }\end{array}$ & $\begin{array}{l}\text { SCL- } 90 \text { were used to identify } 55 \\
\text { subjects with a high risk of suffering } \\
\text { from the depression group, and the } \\
\text { rest are in the control group. The VR } \\
\text { scenes were obtained from third party } \\
\text { sources as a trigger. EEG and ECG } \\
\text { were recorded during the VR session. }\end{array}$ & $\begin{array}{l}\text { Correlation analysis indicated that the } \\
\text { alpha frequency band of the right } \\
\text { forehead in the resting state was } \\
\text { positively related to depression } \\
\text { symptoms, so was the alpha frequency } \\
\text { band of the first HVHA VR scene. }\end{array}$ & $\begin{array}{l}\text { The condition group } \\
\text { is identified as high } \\
\text { risking students, not } \\
\text { clinically diagnosed. } \\
\text { Jadad scale = } 3\end{array}$ \\
\hline$[36]$ & $\begin{array}{l}\text { The study proposed a } \\
\text { personalized VR depression } \\
\text { diagnosis scene and interactive } \\
\text { models for depression } \\
\text { assessment. Designs include the } \\
\text { space design, role design, } \\
\text { iterative technology, audio } \\
\text { material design, and interface } \\
\text { design. }\end{array}$ & $\begin{array}{l}\mathrm{N}=10 \\
5 \text { males and } \\
5 \text { females }\end{array}$ & $\begin{array}{l}\text { A preliminary experiment, the subject } \\
\text { was asked to fill the user experience } \\
\text { questionnaire after a test was } \\
\text { conducted using the design VR } \\
\text { depression diagnosis system. }\end{array}$ & $\begin{array}{l}\text { The experimental results generally show } \\
\text { good feedback on user experience. The } \\
\text { visibility of the doctor's position obtains } \\
\text { the highest score. The lowest is the } \\
\text { naturalness of the scene, mainly affected } \\
\text { by the coordination between the human } \\
\text { doctor and the virtual scene. }\end{array}$ & $\begin{array}{l}\text { No justification of } \\
\text { the efficacy of the } \\
\text { diagnosis, but focus } \\
\text { more on UX design } \\
\text { and satisfaction. } \\
\text { Jadad scale }=1\end{array}$ \\
\hline [37] & $\begin{array}{l}\text { A framework was proposed for } \\
\text { detecting depression in cancer } \\
\text { patients using prosodic and } \\
\text { statistical features extracted by } \\
\text { speech, while chatting with a } \\
\text { virtual coach. }\end{array}$ & $\begin{array}{l}\text { No Clinical } \\
\text { trial }\end{array}$ & $\begin{array}{l}\text { At first, a training set made of cancer } \\
\text { patients is developed; then a user's } \\
\text { speech signal is recorded and is } \\
\text { processed to generate a feature } \\
\text { vector. The user can be classified in } \\
\text { one of the depression scales. }\end{array}$ & $\begin{array}{l}\text { Only the methods were proposed, no } \\
\text { experiment or effectiveness test data. }\end{array}$ & $\begin{array}{l}\text { AR is used to assist } \\
\text { doctors in classifying } \\
\text { depression scale. } \\
\text { Jadad scale }=1\end{array}$ \\
\hline
\end{tabular}




\subsection{Classification of Study Quality}

Jadad Scale [38] was performed to assess the quality of the identified articles. Most of the studies are in the preliminary stages since VR is still gaining popularity in this area. The average Jadad Scale score is 2.6 out of 5 . Although the average score is not high, it does not necessarily indicate that the articles are not of high quality. The value 2.6 was generated because only five studies were identified. However, the preliminary experiments and pilot data reported in these studies are valuable and worth reviewing in detail to provide directions for future work.

\subsection{Study Characteristics}

Table 1, Summary of Findings, summarizes the characteristics of included studies: Methods, Participants, Interventions, Outcomes, and Notes, by following Cochrane Review Guidelines [39]. Jadad Scale scores are in the "Notes" column.

Among the five studies, one uses AR to design a virtual coach [37] who can simulate the interview and analyze the speech signal of the users to assist real doctors in classifying the severity of depression. Two other studies use VR with the same strategy: simulating the interview session with a virtual interviewer $[33,36]$. The SimSensei kiosk study [33] monitors both nonverbal and verbal behaviors of the subjects, and uses only verbal answers to determine the depression level. While both studies assessed the users' feeling of interaction with the system, they did not report the targeted clinical accuracy of detection. Another approach makes use of affective computing by analyzing the electroencephalogram (EEG) and electrocardiogram signals recorded during VR stimulus [35]. The difference of the beta frequency band of the EEG was found in the preliminary test to be significantly different between the experimental group and the control group, which indicates EEG could be a potential indicator for detecting depression. The VR scenes in this study were obtained from third-party providers. Only discrete environmental scenes, such as "farm with animals," "dark basement," "outer space," and a few more were presented. In the only study of a dedicated interactive game developed using VR [34], subjects need to complete a spatial memory navigation task and a spatial working memory task. The outcome of the tasks were used to investigate the memory difference and navigation aspects between the depression group and the control group. It shows that by interacting with users' spatial memory and navigation skills using VR, depression deficits can be detected.

\subsection{Key Findings}

While VR in assisting depression detection has been increasingly recognized, the design of interactive VR games for this purpose falls behind. The studies show that the standalone simulation of interview sessions in VR can encourage patients to disclose their symptoms to some degree and increase the effectiveness of diagnosis as a result. However, the design does not make full use of the advantages of VR applications. The study [34] shows that an interactive VR game has the potential to precisely target the cognitive deficits of depressed patients; hence it can detect depression with high testretest reliability. Another study [35] shows that immersion and the presence of VR can affect the user's emotions and help the diagnosis by recognizing the triggered emotion patterns. These studies demonstrate the potential of interactive VR games in depression detection. Future studies in content designs and measurements will strengthen our understanding of this research area.

\subsection{Extended Review}

Since only five studies were found and identified as qualified in using VR to detect depression, we decided to extend the review and investigated other approaches proposed to assist depression detection in the past decade, especially computer-aided methodologies. Table 2 summarizes tool-assisted data collection methods for pattern detection. Table 3 summarizes the features being used in VR experiments to detect patterns or partial symptoms of depression in addition to the traditional questionnaires.

Table 2. Tool-assisted Methods

\begin{tabular}{|l|l|}
\hline \multicolumn{2}{|l|}{ Tool-Assisted Methods } \\
\hline Smartphones & {$[40,41]$} \\
\hline Wearable devices & {$[42,43]$} \\
\hline Game-based & {$[42,44,45,46]$} \\
\hline Social Media & {$[45,47,48,49,50]$} \\
\hline VR & {$[33,34,35,36,37]$} \\
\hline
\end{tabular}

Table 3. Effective features

\begin{tabular}{|l|l|}
\hline Features \\
\hline Body movement & {$[42,50,51,52,53,54]$} \\
\hline Facial/Video & {$[52,54,55,56,57]$} \\
\hline Vocal/Speech & {$[45,56,58,59,60]$} \\
\hline Social/Cultural analysis & {$[4,61]$} \\
\hline Brain activity signals & {$[42,62]$} \\
\hline Metabolomic data mining & {$[63]$} \\
\hline Gaming Motivation Scale & {$[46]$} \\
\hline Survey/Rating & {$[44,64]$} \\
\hline Memory/Navigation & {$[34]$} \\
\hline
\end{tabular}


Table 2 shows that emerging technologies can assist traditional psychiatric and psychological approaches for the depression diagnosis. Web mining on social media can analyze user inputs from smartphones, wearable devices, and VR, therefore it can effectively interact with users to collect more dynamic information for better assessment. Furthermore, integrating two or more devices, such as a wristband with a VR headset, into one system could be an innovative approach. It will be able to take advantage of multiple emerging technologies and reduce or even eliminate their respective shortcomings for more accurate detection.

Table 3 shows the features utilized for training the models in differentiating patterns in depressed patients and healthy individuals. Many of the patterns can be collected by personal devices, such as smartphones and wearable devices. This type of integrated system has great potential as it is both portable and affordable.

\section{Discussion}

Based on our review of the prior studies in depression detection in the past ten years, we propose a multimodal framework that combines traditional survey-based assessments with additional measurements that can be obtained from VR games. Figure 2 depicts such a system with an interactive VR session as a centerpiece. A game core and four assessment modules with the blocks representing optional features in this detection system.

The core VR game: The VR game will be designed to address one or more deficits in the key depression indicators, such as motion retardation, memory loss, cognitive impairment, and uncontrollable emotions. The VR game will have a central interactive narrative with several minigames that provide different tasks for the targeted deficits.

Pre-assessment module: This module, happening before the VR session, will make use of the traditional pre- and post- measurements. Commonly used depression scales, short conversations, and gaming motivation scales can be used to pre-screen the subjects.

Behavioral analysis module: During the VR session, the subject's behavioral reactions to different game events will be captured by smartphones and ingame performance measures. Evidence of depression can be captured by analyzing the subject's body movements such as head pose and eye gaze, facial expressions, verbal responses, reaction time to game tasks, the completion of motor skills, navigations, and more, as listed in Table 2.

Affective computing module: Sensors or wearable devices will be attached to the subjects to monitor and record the emotional reactivity of subjects during the game session. Psychophysiological signals such as galvanic skin response, heart rate variability, respiration rate, brain electric activity, blood pressure, and temperature, are used to assess users' emotions that are elicited or amplified by the game scenes.

Post-assessment module: After the VR session, the system can choose a suitable fusion method to integrate the results of different techniques and then either compare them with existing benchmarks or construct cloud databases. Building online databases from more studies with the same topic will benefit the research community in this field.

Implementation of this framework does not have to include every block as described above. Instead, a selection of several features can serve the purpose. The selection will be based on device availability, proper fusion, and cross-verification methods. This strategy will lead to effective detection with accessibility.

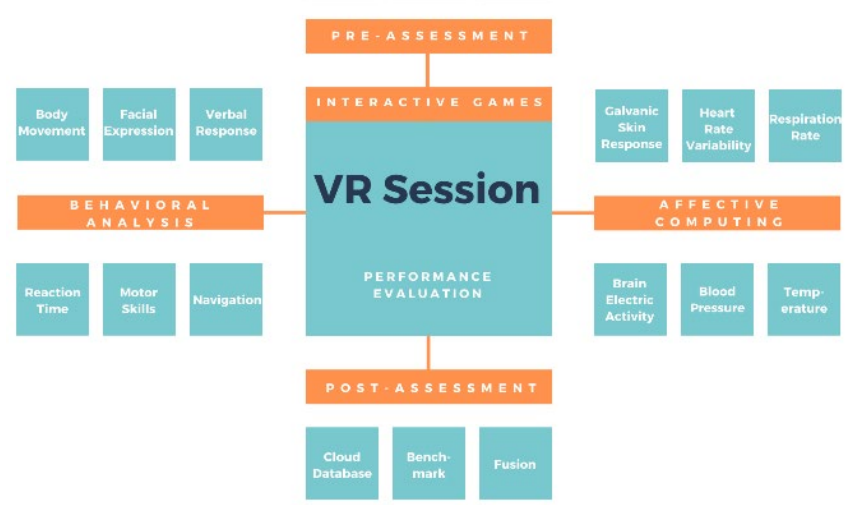

Figure 2. Multimodal detection system

\section{Conclusions and Future Work}

In this paper, we reviewed prior studies to find out how virtual reality has been applied in early depression detection. We also extended the review to explore more technology-based methods for depression detection. Finally, we proposed a multimodal framework with possible methods that work with VR games. This framework uses customizable approaches with portable devices for depression detection. We wish to encourage researchers to study and develop more game-based technologies applying the theories in psychology and psychiatry. We also hope that the research community will find more options to help the patients in more flexible and relaxing environments by using and developing game-based technologies.

For the next step, we plan to implement a customized version of the proposed multimodal system. Psychological signals will be detected, and in-game performance data will be recorded and analyzed to 
assess users' mental capacity. By taking advantage of the presence and immersion of the VR environment, users' psychomotor retardation and memory loss under affected emotion can be captured. As a result, designing and using VR games will lead to the construction of an extendable system with the potential of combining with selective modules for detecting depression and facilitating recovery.

\section{References}

[1] Wan, W., "The coronavirus pandemic is pushing America into a mental health crisis", Washington Post, 2020. https://www.washingtonpost.com/health/2020/05/04/mentalhealth-coronavirus/

[2] Brooks, S.K., R.K. Webster, L.E. Smith, L. Woodland, S.Wessely, N. Greeberg, and G.J. Rubin, "The psychological impact of quarantine and how to reduce it: rapid review of the evidence", Lancet (London, England) 395(10227), 2020, pp. 912-920.

[3] Shigemura, J., R.J. Ursano, J.C. Morganstein, M. Kurosawa, and D.M. Benedek, "Public responses to the novel 2019 coronavirus (2019-nCoV) in Japan: Mental health consequences and target populations", Psychiatry and Clinical Neurosciences 74(4), 2020, pp. 281-282.

[4] Cacheda, F., D. Fernandez, F.J. Novoa, and V. Carneiro, "Early Detection of Depression: Social Network Analysis and Random Forest Techniques", Journal of Medical Internet Research 21(6), 2019, pp. e12554.

[5] Cacheda, F., D. Fernandez, F.J. Novoa, and V. Carneiro, "Early Detection of Depression: Social Network Analysis and Random Forest Techniques", Journal of Medical Internet Research 21(6), 2019, pp. e12554.

[6] Halfin, A., "Depression: the benefits of early and appropriate treatment", The American Journal of Managed Care 13(4 Suppl), 2007, pp. S92-97.

[7] Picardi, A., I. Lega, L. Tarsitani, et al., "A randomised controlled trial of the effectiveness of a program for early detection and treatment of depression in primary care", Journal of Affective Disorders 198, 2016, pp. 96-101. [8] Maples-Keller, J.L., B.E. Bunnell, S.-J. Kim, and B.O. Rothbaum, "The use of virtual reality technology in the treatment of anxiety and other psychiatric disorders", Harvard review of psychiatry 25(3), 2017, pp. 103-113.

[9] Ose, S.O., H. Færevik, J. Kaasbøll, et al., "Exploring the Potential for Use of Virtual Reality Technology in the Treatment of Severe Mental Illness Among Adults in MidNorway: Collaborative Research Between Clinicians and Researchers", JMIR Formative Research 3(2), 2019.

[10] Park, M.J., D.J. Kim, U. Lee, E.J. Na, and H.J. Jeon, “A Literature Overview of Virtual Reality (VR) in Treatment of Psychiatric Disorders: Recent Advances and Limitations", Frontiers in Psychiatry 10, 2019.

[11] Garland, J.E., "Early detection of depression in young and elderly people | British Columbia Medical Journal", 44(9), 2012, pp. 469-472.

[12] Lindner Center of Hope, U.C.H., “Adolescent Depression: Important to Detect and Treat Early $\mid$ Lindner Center of HOPE", 2012. https://lindnercenterofhope.org/blog/adolescent-depressionimportant-to-detect-and-treat-early/

[13] Baños, R.M., C. Botella, M. Alcañiz, V. Liaño, B. Guerrero, and B. Rey, "Immersion and Emotion: Their Impact on the Sense of Presence", CyberPsychology \& Behavior 7(6), 2004, pp. 734-741.

[14] Freeman, D., P. Haselton, J. Freeman, et al., “Automated psychological therapy using immersive virtual reality for treatment of fear of heights: a single-blind, parallel-group, randomised controlled trial", The Lancet Psychiatry 5(8), 2018, pp. 625-632.

[15] Halabi, O., S.A. El-Seoud, J. Alja'am, H. Alpona, M. Al-Hemadi, and D. Al-Hassan, "Design of Immersive Virtual Reality System to Improve Communication Skills in Individuals with Autism", International Journal of Emerging Technologies in Learning (iJET) 12(05), 2017, pp. 50-64. [16] Li, Y., "Designing interactive virtual environments with feedback in health applications.", Electronic Theses and Dissertations, 2018.

[17] Riva, G., "Virtual reality: an experiential tool for clinical psychology", British Journal of Guidance \& Counselling 37(3), 2009, pp. 337-345.

[18] Bohil, C.J., B. Alicea, and F.A. Biocca, "Virtual reality in neuroscience research and therapy", Nature Reviews Neuroscience 12(12), 2011, pp. 752-762.

[19] Li, Y., A.S. Elmaghraby, and E.M. Sokhadze, "Designing immersive affective environments with biofeedback", 2015 Computer Games: AI, Animation, Mobile, Multimedia, Educational and Serious Games (CGAMES), (2015), 73-77.

[20] Juan, M.-M., and L.-A. Magí, "Using Agents in Mental Health: A System to Support the Remote Treatment of Major Depression", Frontiers in Artificial Intelligence and Applications, 2010, pp. 101-110.

[21] Newman, M.G., L.E. Szkodny, S.J. Llera, and A. Przeworski, "A review of technology-assisted self-help and minimal contact therapies for anxiety and depression: Is human contact necessary for therapeutic efficacy?", Clinical Psychology Review 31(1), 2011, pp. 89-103.

[22] Preschl, B., B. Wagner, S. Forstmeier, and A. Maercker, "E-health interventions for depression, anxiety disorders, dementia, and other disorders in old age: A review", Journal of CyberTherapy and Rehabilitation 4, 2011, pp. 371-385.

[23] Zeng, N., Z. Pope, J.E. Lee, and Z. Gao, "Virtual Reality Exercise for Anxiety and Depression: A Preliminary Review of Current Research in an Emerging Field", Journal of Clinical Medicine 7(3), 2018, pp. 42.

[24] "NIMH » Mental Health Information » Health Topics » Depression”, 2018.

https://www.nimh.nih.gov/health/topics/depression/index.sht $\mathrm{ml}$

[25] Segrin, C., "Social skills deficits associated with depression", Clinical Psychology Review 20(3), 2000, pp. 379-403.

[26] Ravnkilde, B., P. Videbech, K. Clemmensen, A. Egander, N.A. Rasmussen, and R. Rosenberg, "Cognitive deficits in major depression", Scandinavian Journal of Psychology 43(3), 2002, pp. 239-251.

[27] Degl'Innocenti, A., H. Ågren, and L. Bäckman, "Executive deficits in major depression", Acta Psychiatrica Scandinavica 97(3), 1998, pp. 182-188. 
[28] Brand, A.N., J. Jolles, and C. Gispen-de Wied, "Recall and recognition memory deficits in depression", Journal of Affective Disorders 25(1), 1992, pp. 77-86.

[29] Morales, M., S. Scherer, and R. Levitan, "A Crossmodal Review of Indicators for Depression Detection Systems", Proceedings of the Fourth Workshop on Computational Linguistics and Clinical Psychology - From Linguistic Signal to Clinical Reality, Association for Computational Linguistics (2017), 1-12.

[30] Riedl, M.O., and V. Bulitko, "Interactive Narrative: An Intelligent Systems Approach”, AI Magazine 34(1), 2013, pp. 67-67.

[31] Liberati, A., D.G. Altman, J. Tetzlaff, et al., "The PRISMA Statement for Reporting Systematic Reviews and Meta-Analyses of Studies That Evaluate Health Care Interventions: Explanation and Elaboration", PLOS Medicine 6(7), 2009, pp. e1000100.

[32] Dybsky, D., "The History of Virtual Reality: Ultimate Guide. Part 2 | TESLASUIT blog", TESLASUIT, 2017. https://teslasuit.io/blog/history-virtual-reality-ultimate-guidepart-2/

[33] DeVault, D., R. Artstein, G. Benn, et al., "SimSensei kiosk: a virtual human interviewer for healthcare decision support", Proceedings of the 2014 international conference on Autonomous agents and multi-agent systems, International Foundation for Autonomous Agents and Multiagent Systems (2014), 1061-1068.

[34] Gould, N.F., M.K. Holmes, B.D. Fantie, et al., "Performance on a Virtual Reality Spatial Memory Navigation Task in Depressed Patients", American Journal of Psychiatry 164(3), 2007, pp. 516-519.

[35] Liang, G., Y. Li, D. Liao, H. Hu, Y. Zhang, and X. Xu, "The Relationship between EEG and Depression under Induced Emotions Using VR Scenes”, 2019 IEEE MTT-S International Microwave Biomedical Conference (IMBioC), IEEE (2019).

[36] Liao, D., L. Shu, Y. Huang, J. Yang, and X. Xu, "Scenes Design in Virtual Reality for Depression Assessment", Virtual, Augmented and Mixed Reality: Applications in Health, Cultural Heritage, and Industry, Springer International Publishing (20180602), 116-125.

[37] Roniotis, A., H. Kondylakis, and M. Tsiknakis, "Detection and Management of Depression in Cancer Patients Using Augmented Reality Technologies, Multimodal Signal Processing and Persuasive Interfaces", 2017 IEEE 30th International Symposium on Computer-Based Medical Systems (CBMS), (2017), 751-752.

[38] "Appendix: Jadad Scale for Reporting Randomized Controlled Trials", In Evidence-based Obstetric Anesthesia. John Wiley \& Sons, Ltd, 2007, 237-238.

[39] Higgins, J., J. Thomas, J. Chandler, et al., Cochrane Handbook for Systematic Reviews of Interventions. 2nd Edition, Chichester (UK): John Wiley \& Sons, 2019. [40] Basco, M.R., M. Kyrarini, and F.S. Makedon, "Personal Devices and Smartphone Applications for Detection of Depression”, Psychiatric Annals 50(6), 2020, pp. 255-259. [41] Saeb, S., E.G. Lattie, K.P. Kording, and D.C. Mohr, "Mobile Phone Detection of Semantic Location and Its Relationship to Depression and Anxiety", JMIR mHealth and uHealth 5(8), 2017, pp. e112.
[42] Maskeliūnas, R., T. Blažauskas, and R. Damaševičius, "Depression Behavior Detection Model Based on Participation in Serious Games", In Rough Sets. Springer International Publishing, 2017, 423-434.

[43] Mengesha, T., "ICT-based bracelet for early detection of depression", 2016. http://jultika.oulu.fi/files/nbnfioulu201605221847.pdf

[44] Bartgis, J., and G. Albright, "Online role-play simulations with emotionally responsive avatars for the early detection of Native youth psychological distress, including depression and suicidal ideation", American Indian and Alaska Native Mental Health Research (Online) 23(2), 2016, pp. 1-27.

[45] Mok, W.T., R. Sing, X. Jiang, and S.L. See, "Proposal of a depression detector", Signal and Information Processing Association Annual Summit and Conference (APSIPA), 2014 Asia-Pacific, IEEE (2014), 1-5.

[46] Peracchia, S., F. Presaghi, and G. Curcio, "Pathologic Use of Video Games and Motivation: Can the Gaming Motivation Scale (GAMS) Predict Depression and Trait Anxiety?", International Journal of Environmental Research and Public Health 16(6), 2019, pp. 1008.

[47] Burdisso, S.G., M. Errecalde, and M. Montes-y-Gómez, "A text classification framework for simple and effective early depression detection over social media streams", Expert Systems with Applications 133, 2019, pp. 182-197.

[48] Cvetković, J., and M. Cvetković, “On community detection in complex networks based on different training algorithms: A case study on prediction of depression of internet addiction", Physica A: Statistical Mechanics and its Applications 523, 2019, pp. 1161-1170.

[49] Gui, T., L. Zhu, Q. Zhang, et al., "Cooperative Multimodal Approach to Depression Detection in Twitter", Proceedings of the AAAI Conference on Artificial Intelligence 33(01), 2019, pp. 110-117.

[50] Wang, Y.-T., H.-H. Huang, and H.-H. Chen, “A Neural Network Approach to Early Risk Detection of Depression and Anorexia on Social Media Text", pp. 8.

[51] Alghowinem, S., R. Goecke, M. Wagner, G. Parkerx, and M. Breakspear, "Head Pose and Movement Analysis as an Indicator of Depression”, 2013 Humaine Association Conference on Affective Computing and Intelligent Interaction, (2013), 283-288.

[52] Holt, R.A., S. Jones, and G. Washington, "Measuring Stress, Anxiety, and Depression in PTSD Sufferers Using Micro-Movements from Video", 2016 IEEE International Conference on Healthcare Informatics, ieeexplore.ieee.org (2016).

[53] Joshi, J., A. Dhall, R. Goecke, and J.F. Cohn, "Relative Body Parts Movement for Automatic Depression Analysis", 2013 Humaine Association Conference on Affective Computing and Intelligent Interaction, (2013), 492-497. [54] Joshi, J., R. Goecke, G. Parker, and M. Breakspear, "Can body expressions contribute to automatic depression analysis?", 2013 10th IEEE International Conference and Workshops on Automatic Face and Gesture Recognition (FG), (2013), 1-7.

[55] Girard, J.M., J.F. Cohn, M.H. Mahoor, S. Mavadati, and D.P. Rosenwald, "Social Risk and Depression: Evidence from Manual and Automatic Facial Expression Analysis", Proceedings of the ... International Conference on Automatic 
Face and Gesture Recognition. International Conference on Automatic Face and Gesture Recognition, 2013, pp. 1-8.

[56] Joshi, J., R. Goecke, S. Alghowinem, et al., "Multimodal assistive technologies for depression diagnosis and monitoring", Journal on Multimodal User Interfaces 7(3), 2013, pp. 217-228.

[57] Venkataraman, D., and N.S. Parameswaran, "Extraction of Facial Features for Depression Detection among Students", International Journal of Pure and Applied Mathematics 118, 2018, pp. 455-462.

[58] Rodrigues Makiuchi, M., T. Warnita, K. Uto, and K. Shinoda, "Multimodal Fusion of BERT-CNN and Gated CNN Representations for Depression Detection", Proceedings of the 9th International on Audio/Visual Emotion Challenge and Workshop, Association for Computing Machinery (2019), 55-63.

[59] Smith, M., B.J. Dietrich, E. Bai, and H.J. Bockholt, "Vocal pattern detection of depression among older adults", International Journal of Mental Health Nursing 29(3), 2020, pp. $440-449$.

[60] Sturim, D., P. Torres-Carrasquillo, T. Quatieri, N. Malyska, and A. McCree, "Automatic Detection of
Depression in Speech Using Gaussian Mixture Modeling with Factor Analysis.", Proceedings of the Annual Conference of the International Speech Communication Association, INTERSPEECH, (2011), 2981-2984.

[61] Kohrt, B.A., N.P. Luitel, P. Acharya, and M.J.D. Jordans, "Detection of depression in low resource settings: validation of the Patient Health Questionnaire (PHQ-9) and cultural concepts of distress in Nepal”, BMC Psychiatry 16(1), 2016, pp. 58.

[62] Shin, D., Y. Nam, D. Shin, and D. Shin, "Real-Time Human Depression Diagnosis System Using Brain Wave Analysis", Advanced Multimedia and Ubiquitous Engineering, Springer (2017), 412-418.

[63] Chovancova, O., J. Rabcan, J. Kostolny, and D. Macekova, "Human Reliability Evaluation through Analysis of Depression Prediction based on Metabolomic Data", 2019 10th International Conference on Dependable Systems, Services and Technologies (DESSERT), IEEE (2019). [64] Ansari, H., A. Vijayvergia, and K. Kumar, "DCRHMM: Depression detection based on Content Rating using Hidden Markov Model", 2018 Conference on Information and Communication Technology (CICT), (2018), 1-6. 